\title{
Trend in laryngeal cancer, mortality and survival rate in Iran
}

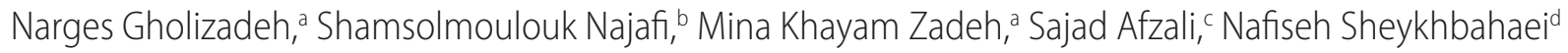

\author{
aDepartment of Oral \& Maxillofacial Medicine, School of Dentistry, International Campus, Tehran University of Medical Science, Tehran, Iran. \\ ${ }^{b}$ Dental Research Center, Tehran University of Medical Science, Tehran, Iran. \\ 'School of Dentistry, Tehran University of Medical Science, Tehran, Iran. \\ ${ }^{d}$ Research Center for Caries Prevention, Dentistry Research Institute, Tehran University of Medical Sciences, Tehran, Iran. \\ Correspondence to Nafiseh Sheykhbahaei (email: dsheykhbahaei@gmail.com). \\ (Submitted: 18 October 2017 - Revised version received: 27 October 2017 - Accepted: 20 November 2017 - Published online: 26 March 2018)
}

\begin{abstract}
Objective Among the noncommunicable disease, cancer is one of the highest load and charge that lead to disability and death. The goal of this study was to determine the important epidemiologic factors that effect on survival rate of patients with laryngeal cancer.

Methods From 307 cases, 136 (2009-2012) that their follow-up was possible by phone or postal address were included in the study. Data collections were performed from the questionnaire. The data were analyzed by SPSS version 20. The Kaplan-Meier survival curves were utilized and, moreover, the corresponding influential factors were examined by using the Cox regression test.

Results The most widely used method for the treatment was a combination of three methods of surgery, radiation therapy, and chemotherapy. The overall 5-year survival rate was $47.28 \%$. The age and regular follow up had a statistically significant relationship with the survival rate $(P=0.02,0.03)$.

Conclusion The survival rate was lower in older patients. Patient and professional delays were high, which need more attention for improving the quality of life.

Keywords laryngeal neoplasms, mortality, survival rate, trends
\end{abstract}

\section{Introduction}

Among the noncommunicable diseases, cancer is one of the highest load and charge that lead to disability and death. According to WHO report, the rate of death caused by cancer, precede from cardiovascular diseases. ${ }^{1}$ A common feature among all cancer cells is the loss of responsiveness to the normal growth control. Physical and emotional harm caused by cancer is greater than mortality rate. ${ }^{2}$ Either health care condition or quality of life in cancer patients significantly affected by elevation cancer incidence rate. Head and neck cancers are a group of cancers in the variant area containing the lip, oral cavity, pharynx, hypopharynx, and the larynx. ${ }^{1}$ Head and neck cancer is the sixth common cancer in the world, and among these, the oral cavity is the most commonly encountered place. ${ }^{3}$ In the ranking of the common causes of men's mortality in the worldwide, the oral cavity cancer is among the top 10 causes. ${ }^{4}$ More than $90 \%$ of the oral cavity cancers comprising squamous cell carcinoma (SCC) that originates from the epithelial lining of oral cavity, including tongue, floor of mouth and lips. ${ }^{5}$ Laryngeal cancer is the second most common cancer in the upper aero digestive system. According to previous studies, $30-40 \%$ of all malignant tumors of the head and neck as well as $1.5-2 \%$ of all malignancies are associated with laryngeal cancer. In addition, 85-98\% of laryngeal cancers are SCC. The most common sites of tumor involvement are glottic, supraglottic or both parts. ${ }^{6}$ Clinical presentations can be as ulceration or exophytic mass. Histological examination from biopsied specimens is mandatory to definitive diagnosis. High diversity in clinical manifestation and insufficient attention and follow up of potentially malignant lesions lead to delay in diagnosis. ${ }^{7}$ The most common risk factors for head and neck SCC include individual habits such as tobacco and alcohol use. In the last 10 years, smoking has fallen by $20 \%$ in adult. This despite the fact that alcohol consumption has increased among men and women. Also, association between HPV infection and head and neck cancer has been established. ${ }^{8}$ Environmental factors, such as poor oral hygiene, denture use, bleeding gums, infrequent dental visits, ${ }^{9}$ inappropriate diets, especially lack of fruits and vegetables, lack of physical activity, and obesity seems that play more important role than congenital and genetical factors in etiology of cancer. Contamination by carcinogenic agents, such as asbestos, polycyclic aromatic hydrocarbons, and dust, are also among the ingredients that increase the risk of these cancers. ${ }^{10,11}$ According to the WHO reports, the highest age-standardized rate of such head and neck cancers is found in the Southeast Asia region. ${ }^{1}$ In Iran, 70,000 new cases of cancer occur per year and over 30,000 people die annually from cancer. ${ }^{12}$ According to recent evidence, the 5 -year survival rate of 23 types of cancer has increased among 24 types of cancer over the past 25 years, but only the survival rate of laryngeal cancer has declined over these years. Although the main cause of this decrease in survival rate are not well known, during this period, sharply decrease in surgical procedures and increase in the use of nonsurgical methods, such as chemotherapy and radiotherapy have been observed..$^{13}$ In addition, due to the improvement of health care level, the average age of the general population and people with cancer are increased. Other suggestive factors are dietary changes and increased alcohol use. The changes in socioeconomic status of individuals are the possible causes for reducing the survival of patients with laryngeal cancer. ${ }^{13,14}$ In recent years, changes in risk factors exposure, diagnostics, staging, and treatment methods caused to significant changes in incidence, mortality and survival rate. Policy determination to improve prevention, treatment, and supervision care for these patients need to evaluate the trends in head and neck cancer. ${ }^{15}$

Due to the relatively high prevalence of head and neck cancer especially laryngeal carcinoma in the Southeast Asian 
regions like Iran, and the lack of accurate statistics about the trend of these cancers in this area, we evaluated the relationship between clinical and demographic factors and treatment type with improved survival rate among patients with laryngeal cancer, using data collected by the Cancer Registry Center of Ministry of Health and Medical Education for patients diagnosed from 2009 to 2012 .

\section{Materials and Methods}

In this retrospective cross-sectional study, for data collection, we used documents related to laryngeal cancer patients from 2009 to 2012 in Cancer Department of the Ministry of Health of the Islamic Republic of Iran. From a total of 307 medical records with a histopathological diagnosis of laryngeal squamous cell carcinoma, 136 cases with contact information or mailing address for correspondence were included in the study. Since 2003, all new cases of cancer detection in Iran have been referred to cancer registry centers through the Ministry of Health's designated forms from pathology labs. These forms include demographic characteristics, pathological factors of the disease and diagnostic times. The location of the lesion, the degree of differentiation, and degree of invasive are also included with the special code in these cases. In this study, a checklist containing two categories of questions was prepared to collect and categorize information about patients. The first group questions were completed based on the information in Cancer Registration Center forms. The second part of the questions in checklist was completed by telephone conversations by patients or relatives, including survival information, types of treatments and delayed time. In the case of non-responding to a telephone call after three times of contact with a 2-week interval, we used the postal service to obtain the patient information, and if the person did not respond, their information extracted from his medical records available in the Cancer Institute of Imam Khomeini Hospital. In the case of dead patients, the cause of death was asked from relatives of patients, and two death groups were classified as death due to complications of cancer and other causes such as accidents or heart attack. Patient delay (time interval from the occurrence of disease symptoms to the first patient referral), and professional delay (time interval from the first patient referral to diagnosis) evaluated in the two groups, less than 4 weeks and more than 4 weeks.
The study was approved by the Ethics Committee of Tehran University of Medical Science.

The collected data were analyzed using SPSS20 software and tables and figures were presented. The survival rate was investigated with Kaplan-Meier curve, the effect of the studied factors on the survival rate with simple and multivariate Cox regression test. The statistical significance level was considered to be $P<0.05$.

\section{Results}

\section{Patient Demographics and Clinical Data}

According to present study findings, the prevalence of laryngeal cancer was 20 times higher in men than in women. The participants consisted of 130 men (95.58\%) and six women (4.42\%), and also $98.42 \%$ of the patients were married.

The mean age of the patients was $60.8,46.3 \%$ of patients equal to or less than 60 years and $53.7 \%$ more than 60 years. The age range of the patients was 28-85 years (Fig. 1). The majority of the cancer patients were in the sixth to eighth decades of their life. The patients were categorized according to the histological grade of the lesions, $41.81 \%$ with completely differentiated lesions, $38.18 \%$ with relatively differentiated lesions and $20.01 \%$ with less differentiated lesions.

\section{Diagnostic Process}

In this study, the results regarding awareness of the patients about their disease showed that $82(74.54 \%)$ found the symptoms of cancer themselves and $16(14.54 \%)$ were aware of their risk of developing cancer by referring to a physician due to other illnesses. The statistical findings of the first referral in this study can be categorized according to whom the patient has referred to for the first time. The first referral of 98 patients $(80.34 \%)$ was to the specialist physician, five $(4.09 \%)$ to the dentist and the rest $(15.57 \%)$ to the general practitioner.

\section{Delay in Cancer Management}

The reasons for the delay among the patients were inaccessibility to health centers (6.7\%), failure to receive appointment (5\%), unable to pay the costs (20\%), and lack of insurance coverage (1.7\%). Others did not provide clear reasons. The professional delay was obtained on average 4.26 weeks.

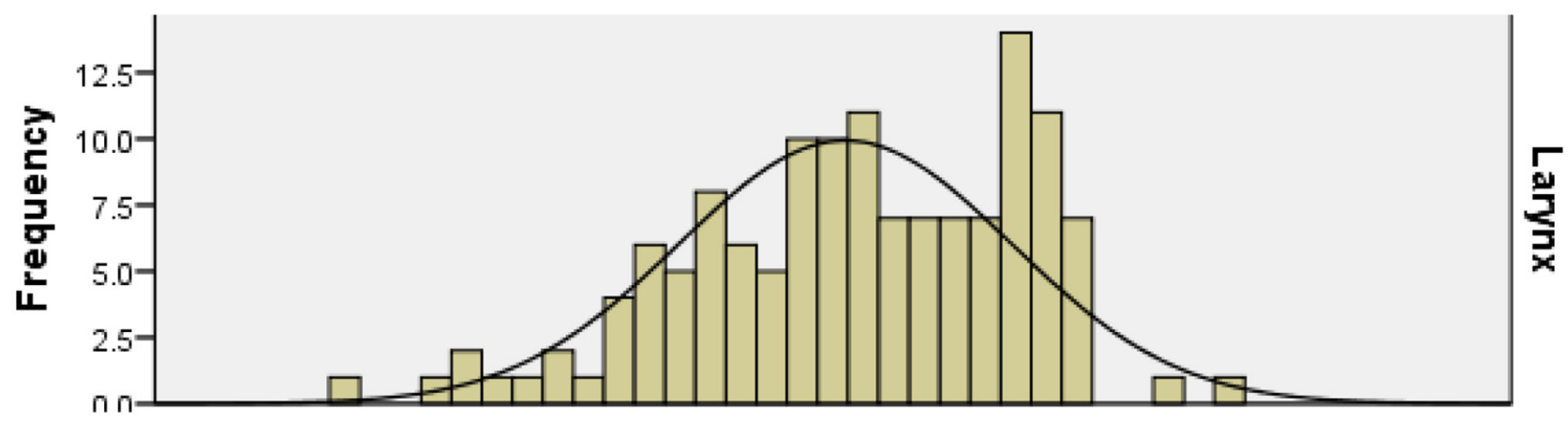

20.00

40.00

60.00

80.00

$100 \cdot .00$

\section{Age at diagnosis (year)}

\section{Fig 1. Histogram of patient's age.}




\section{Treatment Outcomes}

The patients with laryngeal cancers were treated in three ways: surgery, radiotherapy, and chemotherapy. In many cases, the treatment of patients was a combination of two or three methods. Accordingly, the most commonly used treatment (37.5\%) for the patients was the combination of all three treatments. Surgery $12.5 \%$, chemotherapy $6.6 \%$, radiotherapy $5.1 \%$, chemo-radiotherapy $10.3 \%$, chemo-surgery $8.1 \%$, radio-surgery $11 \%$, and $0.7 \%$ of patients without treatment.

\section{Follow-up}

In the periodic follow-up, $56(70 \%)$ patients in the hospital and $21(26.25 \%)$ in the private medical clinic had periodic follow-up, and three (3.75\%) had no follow-up programs. Also, $12.1 \%$ of patients had periodic follow-up under supervision of oral hygienist.

\section{Survival Rate}

The study showed that $62(45.59 \%)$ patients survived during the study but 74 (54.59\%) died. Findings related to the cause of death in the patients indicated that $67(93.03 \%)$ died due to the cancer complications and five (6.95\%) for other causes. According to statistical analysis, the overall 1-year survival rate of these patients was $88.37 \%$ with the standard error of 2.82 , the overall 3-year survival rate was $58.13 \%$ with the standard error of 4.34 and the overall 5 -year survival rate was $47.28 \%$ with the standard error of 4.4 (Table 1).

The statistical analysis showed that the survival rate was higher in women than in men, but the gender-specific had no significant relationship with survival rate $(P=0.41)$. In addition, the survival rate was significantly associated with the age of the patients, so that the survival rate decreased with age $(P>0.001)$. In addition, regular periodic follow-up leads to significant improvement in survival rate $(P<0.03)$. Also, there was no significant difference in survival rate among patients with periodic follow-up by oral hygienists $(P=0.51)$. The patient's delay rate more than 4 weeks was not significantly associated with survival rate $(P=0.37)$. The survival rate also had no significant relationship with the rate of professional delay $(P=0.54)$. The results showed that the survival rate in these patients had no significant relationship with the grade of histological differentiation of tumor $(P=0.35)$. According to the multivariate Cox regression analysis, individual treatments had no significant relationship with survival rate (combination of three methods $P=0.055$, chemo-radiotherapy $P=0.64$ and radiotherapy $P=0.73$ ) (Table 2).

\section{Discussion}

In the present study, we evaluate the effect of patient and tumor characteristics, diagnostic process, treatment outcome,

\begin{tabular}{|c|c|}
\hline Duration (year) & Survival rate \\
\hline 1 & $88.38 \%(\mathrm{SE}=2.82)$ \\
\hline 2 & $68.21 \%(S E=4.09)$ \\
\hline 3 & $58.13 \%(S E=4.34)$ \\
\hline 5 & $47.28 \%(S E=4.4)$ \\
\hline
\end{tabular}

Table 2. Association of studied factors with survival rate among laryngeal cancer patients $(n=136)$

\begin{tabular}{lcc}
\hline Factor & P-value $^{*}$ & $\begin{array}{c}\text { 95\% Confidence } \\
\text { interval }\end{array}$ \\
\hline Age & $P>0.001^{*}$ & $8.12-3.88$ \\
Gender & $P=0.41$ & $2.58-8.38$ \\
Histological grade of the tumor & $P=0.35$ & $2.82-0.09$ \\
Tumor location & 2.58 & $2.88-8.02$ \\
Patient's delay & $P=0.37$ & $2.18-8.89$ \\
Professional delay & $P=0.54$ & $2.81-8.08$ \\
Periodic follow-up & $P=0.03^{*}$ & $8.23-0.89$ \\
Combination three method & 0.055 & $2.11-8.28$ \\
treatment & & $2.80-8.02$ \\
Chemo-radiotherapy & 0.64 & $2.81-8.05$ \\
\hline Radiotherapy & 0.73 &
\end{tabular}

*Significance level was 0.05 .

and follow-up on survival rate in patients with laryngeal cancer in Iran between 2009 and 2012. The results demonstrated significantly higher survival rates in patients under age 60 and regular periodic follow-up. We gathered data from Cancer Registry Center of Ministry of Health and Medical Education and Cancer Institute of Imam Khomeini Hospital.

Our data demonstrate that the overall 5-year survival rate was $47.28 \%$. In the study of Ramroth et al., ${ }^{16}$ the overall 5 -year survival rate of patients was $66 \%$, about $10 \%$ higher than the current study. In the study by Fararouei et al., ${ }^{6}$ the meansurvival rate of the patients was $46.86 \pm 38.66$. Other reported 5 -year survival rates were 56 and $67.6 \% .{ }^{17,18}$ In another study, 1-year survival was reported to be $3.79 \%$ in early-stage patients. ${ }^{19}$ Nachalon et al. ${ }^{20}$ reported the overall survival rate of $30.8 \pm 4.6$ years.

Comparing the survival rate in various studies is very challenging and different, which can be explained by the following reasons: different types of disease and patient selection in studies that may be limited based on specific therapeutic methods, site of the tumor and stage of the tumor, as well as the limited sample size is one of the main factors of varying survival rates in studies. ${ }^{6}$ This study showed a higher prevalence of laryngeal SCC in males so that the number of men was $130(95.58 \%)$ and women were six (4.42\%). Regarding the results of statistical analyses in this study, the gender had no significant relationship with survival rate. Studies in Germany ${ }^{16}$ and Finland ${ }^{21}$ also revealed that men accounted for more than $90 \%$ of the patients. In the study in 2017,6232 out of 250 patients with laryngeal cancer were males. Nachalon et al. ${ }^{20}$ also reported that the ratio of men to women was 2.6/1. Chen and Boffetta ${ }^{14,22}$ demonstrated that survival rate was significantly lower in men than in women, while in other studies, as the results of the current study, this significant relationship

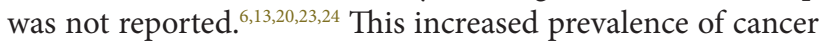
and reduced survival rate in men may be due to higher exposure to risk factors, as well as less attention of men to individual health and more delay in referral to a physician.

We find that more than half of cancer patients were over 60 years of age. The mean age of the patients in the study by Ramroth et al. ${ }^{16}$ was approximately slightly higher (63 years) than the present study. However, the number of people under 60 years old in their study was $38 \%$, which is much less than research in 
Tehran. This may be due to differences in people's lifestyles. They also reported 50\% increase in mortality per decade of aging. In other studies also reported that age increasing was significantly associated with a reduction in survival rate. . $14,23,24^{2}$ They suggested that aging leads to increased comorbidities (such as cardiovascular, respiratory, and renal failure) in the patients. The combination of these two phenomena can be a significant predictor of the mortality rate of the patients. ${ }^{23}$

In our study, the histological grade of the tumor indicated no significant relationship with survival rate $(P=0.35)$. In the study of Fararouei et al., ${ }^{6}$ the tumor grade was introduced an important factor in the survival rate of patients. However, most researchers who evaluated the survival rate, have examined the relationship between tumor stage at the time of diagnosis and survival rate rather than grade. ${ }^{13,20,23,24}$

Similar to the results of this study, two other studies shown that the tumor site did not lead to significant changes in the survival rate of the patients with laryngeal cancer. ${ }^{6,13}$ According to previous reports, glottic region involvement due to hoarseness is commonly diagnosed in the early stages of cancer. However, supraglottic cancer due to the lack of symptoms is usually diagnosed in the more advanced stages of the tumor, which can lead to a reduction in the survival.

Using collected data, the effect of patient and professional delay rates on survival rate of patients was measured. The mean of patient delay was 12.53 almost equivalent 3 months, ranging from 1 week to 1 year (52 weeks). The mean professional delay was about 4.26 weeks ranging from 1 to 39 weeks. One research in 2003 demonstrated that professional delay ( $\geq 12$ months) was statistically significant determinant of survival rate $(P$-value $=0.05)$. While there was no correlation between patient delay and prognosis. ${ }^{21}$ Alho and Teppo, ${ }^{21}$ in 2009 indicated the mean patient delay, 2 months and mean professional delay, 2.1 months. Their results are almost consistent with the present study, but the study in Tehran shows a slightly higher delay in the mean patient and professional delay. As stated above, higher delay can indicate a low awareness of cancer patients or inadequate access to treatment centers for the diagnosis and treatment of cancer, inadequate economic status, insurance coverage or inadequate medical facilities and resources.

In this research, there was no significant difference in the survival rate of patients based on the type of treatment. Similarly, Sabin et al. ${ }^{23}$ reported that the therapeutic method did not significantly increase the survival rate. Although, our results show that in cases where surgery has been used in combination with other treatments, survival rate has increased impressively $(P=0.055)$. Nevertheless, some studies found a significant difference in the type of treatment with survival rate. In all of these studies, it has been shown that the use of surgery alone or in combination with other therapies significantly increases survival of the patients with laryngeal cancer, as well as could lead to lower costs and subsequent problems for patients. ${ }^{6,13,22,24-26}$ The most widely used treatment method for the patients in the present study population was a combination of all three methods of surgery, radiotherapy and chemotherapy. Among the patients with laryngeal cancer, $37.5 \%$ experienced all three treatments. However, in the study by Ramroth et al., ${ }^{16} 19.2 \%$ had all three treatments.

Our findings also confirm that the regular follow-up of the patients by the expert can significantly improve survival rate, although these results were not seen in patients who were under the supervision of oral hygienists. This indicates that the careful examination by physician is very effective in identifying lesion recurrence and early treatment.

\section{Conclusion}

In conclusion, early diagnosis and treatment of disease at an earlier age can be a major factor in increasing the survival rate of the patients with laryngeal cancer. Hence, increasing the level of awareness of patients about warning signs and symptoms, high-quality education for health care workers and hygienists to identify normal from abnormal head and neck structures, improving the economic conditions, access to health systems to reduce costs and delay in cancer management and emphasize the proper implementation of referral and follow up systems for patients should be at the head of community-based health policies.

\section{Acknowledgments}

Thank for friendly cooperation of the Center for the Control and Prevention of Noncommunicable Diseases, Cancer Department of the Ministry of Health of the Islamic Republic of Iran, who helped us in this study.

\section{Conflict of interest}

None.

\section{References}

1. Azimi S, Mortazavi H, Tennant M, Kruger E, Rezaei B, Taheri JB, et al Pattern of the head and the neck cancer in two geographically and socioeconomically different countries. J Orofac Sci. 2017:9:43-47.

2. Sikarwar S, Shrivastava JP, Magnani KK, Shah M, Jain B. "Oral cancer claws clutching the youth" A study of oral cancers at a tertiary care center in central India. IJAR. 2017:3:514-517.

3. Gupta A, Agrawal G, Tiwari S, Verma K, Agrawal R, Choudhary V. Pectoralis major myocutaneous flap in head and neck reconstruction: an interesting experience from central India regional cancer center. Int J Res Med Sci. 2015:3:3065-3068.

4. Ribeiro, IP, Barroso L, Marques F, Santos A, Caramelo F, Julião MJ, et al. Genomic and epigenetic characterization for the comparison of synchronous bilateral tongue squamous cell carcinomas - a case report. Curr Probl Cancer. 2017;41:398-406.

5. Ogmundsdóttir HM, Björnsson J, Holbrook WP. Role of TP53 in the progression of pre-malignant and malignant oral mucosal lesions. A followup study of 144 patients. J Oral Pathol Med. 2009;38:565-571.

6. Fararouei M, Daneshi N, Mohammadianpanah M, Reza Tabatabaei H, ZareBandamiri M, Dianatinasab M. Factors predicting survival in patients with early stage laryngeal cancer: a cohort study between 2000 to 2015. J BUON. 2017:22:996-1003

7. Hingorani DV, Lemieux AJ, Acevedo JR, Glasgow HL, Kedarisetty S, Whitney $M A$, et al. Early detection of squamous cell carcinoma in carcinogen induced oral cancer rodent model by ratiometric activatable cell penetrating peptides. Oral Oncol. 2017:71:156-162.

8. Javadi P, Sharma A, Zahnd WE, Jenkins WD. Evolving disparities in the epidemiology of oral cavity and oropharyngeal cancers. Cancer Causes Control. 2017;28:635-645

9. Hashim D, Sartori S, Brennan P, Curado MP, Wünsch-Filho V, Divaris K, et al. The role of oral hygiene in head and neck cancer: Results from International Head and Neck Cancer Epidemiology (INHANCE) consortium. Ann Oncol. 2016:27:1619-1625

10. Ramroth H, Dietz A, Becher H. Interaction effects and populationattributable risks for smoking and alcohol on laryngeal cancer and 
its subsists: a case-control study from Germany. Methods Inf Med. 2004:43:499-504.

11. Ramroth H, Ahrens W, Dietz A, Becher H. Occupational asbestos exposure as a risk factor for laryngeal carcinoma in a population based case control study from Germany. Am J Ind Med. 2011;54:510-514.

12. Education MoHaM (Ed.). Iranian Annual of National Cancer Registration Report 1387.

13. Petrakos I, Kontzoglou K, Nikolopoulos TP, Papadopoulos O, Kostakis A. Glottic and supraglottic laryngeal cancer: Epidemiology, treatment patterns and survival in 164 patients. J BUON. 2012;17:700-705.

14. Boffetta P, Merletti F, Faggiano F, Migliaretti G, Ferro G, Zanetti R, et al. Prognostic factors and survival of laryngeal cancer patients from Turin, Italy. A population-based study. Am J Epidemiol. 1997;145:1100-1105.

15. van Dijk BA, Brands MT, Geurts SM, Merkx MA, Roodenburg JL. Trends in oral cavity cancer incidence, mortality, survival and treatment in the Netherlands. Int J Cancer. 2016;139:574-583.

16. Ramroth H, Schoeps A, Rudolph E, Dyckhoff G, Plinkert P, Lippert B, et al. Factors predicting survival after diagnosis of laryngeal cancer. Oral Oncol. 2011:47:1154-1158.

17. Chen M-F, Chang JT-C, Tsang NM, Liao C-T, Chen WC. Radiotherapy of earlystage glottic cancer: analysis of factors affecting prognosis. Ann Otol Rhinol Laryngol. 2003;112:904-911.

18. Preuss S, Cramer K, Klussmann J, Eckel HE, Guntinas-Lichius O. Transoral laser surgery for laryngeal cancer: outcome, complications and prognostic factors in 275 patients. Eur J Surg Oncol. 2009;35:235-240.
19. Yu Q, Zhang X, Ji C, Yang H, Gao M, Hong S, et al. Survival analysis of laryngeal carcinoma without laryngectomy, radiotherapy, or chemotherapy. Eur Arch Otorhinolaryngol. 2012;269:2103-2109.

20. Nachalon Y, Cohen O, Alkan U, Shvero J, Popovtzer A. Characteristics and outcome of laryngeal squamous cell carcinoma in young adults. Oncol Lett. 2017;13:1393-1397.

21. Teppo H, Koivunen P, Hyrynkangas K, Alho OP. Diagnostic delays in laryngeal carcinoma: professional diagnostic delay is a strong independent predictor of survival. Head Neck. 2003;25:389-394.

22. Chen AY, Halpern M. Factors predictive of survival in advanced laryngeal cancer. Arch Otolaryngol Head Neck Surg. 2007;133:1270-1276.

23. Sabin SL, Rosenfeld RM, Sundaram K, Har-el G, Lucente FE. The impact of comorbidity and age on survival with laryngeal cancer. Ear Nose Throat J. 1999;78:578, 581-584.

24. Gourin CG, Dy SM, Herbert RJ, Blackford AL, Quon H, Forastiere AA, et al. Treatment, survival, and costs of laryngeal cancer care in the elderly. Laryngoscope. 2014;124:1827-1835.

25. Rudolph E, Dyckhoff G, Becher H, Dietz A, Ramroth H. Effects of tumour stage, comorbidity and therapy on survival of laryngeal cancer patients: a systematic review and a meta-analysis. Eur Arch Otorhinolaryngol. 2011;268:165-179.

26. Francis E, Matar N, Khoueir N, Nassif C, Farah C, Haddad A. T4a laryngeal cancer survival: retrospective institutional analysis and systematic review. Laryngoscope. 2014;124:1618-1623.

This work is licensed under a Creative Commons Attribution-NonCommercial 3.0 Unported License which allows users to read, copy, distribute and make derivative works for non-commercial purposes from the material, as long as the author of the original work is cited properly. 